\title{
0 uso de filmes como recurso pedagógico no ensino de neurofarmacologia
}

Setsuko Noro dos Santos ${ }^{1}$

André Noro $^{2}$

\section{Introdução}

Os recursos audiovisuais têm sido frequentemente utilizados como facilitadores no processo-ensino aprendizagem. A produção de documentários, vídeos e animações para uso específico em sala de aula tem se multiplicado e facilitado a utilização de tais recursos, em especial, por se encontrarem disponibilizados de forma gratuita na internet (Maestrelii, Farrari, 2006).

Outra forma é a utilização de filmes comerciais como recurso pedagógico.

De acordo com Farré et al. (2004, p.1), " filmes comerciais são aqueles produzidos para serem exibidos em salas comerciais ou canais de TV, não sendo feitos para serem utilizados como ferramenta de ensino". São baseados em histórias de ficção ou são livres adaptações de uma história ou fato real. A qualidade dos filmes, a boa atuação dos atores e atrizes envolvidos, e a forma como a história é desenvolvida favorecem a confiabilidade dos espectadores e a aceitabilidade entre os jovens, tornando-os muito convenientes para serem utilizados em atividades de ensino (Farré et al., 2004). Muitos desses filmes estão sendo reconhecidos e utilizados para as discussões em assuntos acadêmicos, sociais, éticos, econômicos e políticos (Toman, Rak, 2000; Berger, Pratt, 1998; Conner, 1996; Anderson, 1992). Na área de Ciências Biológicas e da Saúde, os filmes comerciais têm sido utilizados para auxiliar no ensino de temas relacionados à: ética em pesquisa, Genética, Microbiologia, Psicologia, Psiquiatria, Neurologia e Farmacologia (Gallagher et al., 2011; Guilhem, Diniz, Zicker, 2007; Maestrelli, Ferrari, 2006; Bhagar, 2005; Sierles, 2005; Farré et al., 2004; Lepicard, Fridman, 2003; Crellin, Briones, 1995; Koren, 1993).

Para o ensino da Neurofarmacologia, a utilização de filmes comerciais tem contribuído para a reflexão e entendimento de situações clínicas (Gallagher et al., 2011; Farré et al., 2004; Koren, 1993); entretanto, para a obtenção de maior aproveitamento, é importante que sejam previamente acompanhados de referencial teórico para subsidiar um debate posterior. Para cada aula acompanhada de filme, um planejamento cuidadoso é necessário, desde a sua escolha, que deve estar relacionada com o tema abordado, como, também, pela disponibilidade em locadoras, livrarias, ou fácil localização em livrarias virtuais.

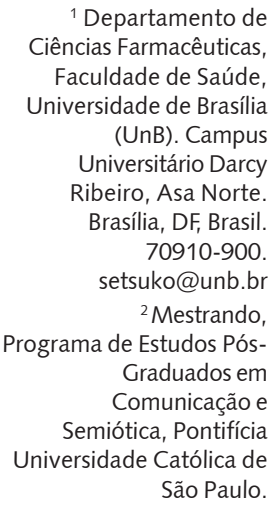

Dartamento de ências Farmacêuticas, Faculdade de Saúde, (UnB). Campus Universitário Darcy Ribeiro, Asa Norte. sília, DF, Brasil. suko@unb.br Estudos PósGraduados em Comunicação e São Paulo. 
O presente trabalho relata uma experiência com a utilização de filmes como ferramenta pedagógica na disciplina neurofarmacologia ofertada para o curso de enfermagem da Universidade de Brasília (UnB). Entende-se, no entanto, que tal recurso pedagógico poderá ser também aplicado em outros cursos da área da saúde, inclusive, em atividade de pós-graduação.

A disciplina está organizada em aulas teóricas, seguidas de apresentação de um caso clínico relacionado ao assunto abordado, com discussão teórica farmacológica para facilitar a assimilação do assunto. Em seguida, um filme é indicado aos alunos, tomando-se o cuidado de escolher aqueles que tratem de determinada patologia, com a terapêutica e cuidados de saúde requeridos. Como a disciplina supramencionada requer um conhecimento prévio de Fisiopatologia, Farmacologia Geral e do conteúdo de doenças psiquiátricas, recomenda-se que seja ofertada para alunos com conhecimentos prévios de tais disciplinas.

Trabalhos publicados, relacionados à utilização de filmes como recurso pedagógico, sugerem que os filmes sejam exibidos em sala de aula, seguidos, posteriormente, por um debate sobre o mesmo (Gallagher et al., 2011; Guilhem, Diniz, Zicker, 2007; Maestrelli, Ferrari, 2006; Hyde, Fife, 2005; Farre et al., 2004). No planejamento aqui adotado, os alunos assistem aos filmes como atividade extraclasse, organizados em grupos pequenos (máximo de cinco alunos), de modo que possam registrar aspectos clínicos e os nomes dos fármacos utilizados ou relatados no filme. Assistir aos filmes fora do ambiente da sala de aula possibilita a ação de pausas e retrocessos para anotações necessárias.

Ao final de cada filme, cada grupo de estudantes prepara um relatório indicando: a provável patologia, os personagens (pacientes) envolvidos, as manifestações clínicas, os fármacos mencionados; descrevendo os aspectos farmacodinâmicos e farmacocinéticos, além dos prováveis efeitos adversos, e, no caso específico do curso de enfermagem, os processos de enfermagem relacionados àquela patologia.

Os relatórios são entregues na aula seguinte, momento em que se dá a discussão sobre o filme assistido. São enfatizados, então, os aspectos clínicos da patologia abordada no filme, relacionando-os aos fármacos citados e seus aspectos farmacológicos. Outros fármacos também utilizados nessa patologia são relembrados durante a discussão, mesmo que não tenham sido citados no filme, analisando-se seus aspectos farmacológicos.

Para a utilização pedagógica de filmes no curso de Neurofarmacologia, sugerem-se alguns passos para facilitar o planejamento e a condução das aulas, bem como alguns filmes já utilizados durante o referido curso. E, ainda, o mesmo recurso pedagógico poderá ser adaptado para o curso de Farmacologia Clínica ou outras disciplinas.

\section{Planejamento da aula}

- Organizar o conteúdo programático do curso, elegendo os temas a serem abordados entre os temas abordados no curso, tem-se: farmacologia da anestesia geral, fármacos ansiolíticos, antidepressivos, antipsicóticos, fármacos usados no tratamento de doenças neurodegenerativas (como, por exemplo, Parkinson e Alzheimer) e farmacologia da dependência e abuso de drogas. $\mathrm{Na}$ condução das aulas, o referencial teórico é um recurso utilizado pelo professor. O conteúdo aborda o conceito da patologia estudada, a fisiopatologia, os aspectos clínicos, mas a ênfase maior recai sobre o tratamento medicamentoso e todos os aspectos relacionados à farmacoterapia, por tratar-se de um curso de Neurofarmacologia.

- Definir os objetivos de cada aula - entre os objetivos constam: a definição da situação/doença, fisiopatologia, aspectos clínicos e terapêuticos, abordando a farmacodinâmica, farmacocinética, interação medicamentosa e efeitos adversos.

- Selecionar o filme a ser utilizado - optar, sempre que possível, por filmes que apresentem proximidade com a vida real ou com os temas a serem discutidos.

- Conhecer o enredo - assistir ao filme antes de indicar aos alunos e verificar sua adequação ao tema que será abordado.

- Preparar um roteiro sobre os principais aspectos a serem analisados - no curso de Neurofarmacologia, o roteiro é importante, uma vez que será solicitado um relatório, além da discussão 
em sala de aula. Caso se opte pela discussão após a exibição do filme, pode-se elaborar um roteiro de questões a serem debatidas após o término do filme, ou iniciar a discussão sem definir previamente o roteiro, a partir de questionamentos dos participantes ou do professor.

\section{Indicação do filme}

Para cada aula, é indicado um filme relacionado com o assunto estudado (ver Quadro 1). Assistir ao filme previamente ao debate compõe a atividade extraclasse. A entrega do relatório e o debate sobre o filme ocorrerão na aula seguinte. Para facilitar a elaboração do relatório, apresenta-se um modelo de roteiro.

Roteiro para elaboração do relatório sobre o filme:

- Tema da aula

- Nome do filme

- Ficha técnica: título original, diretor, produtor, elenco principal, país, ano.

- Breve resumo sobre o enredo do filme: indicar qual a temática (enredo), provável patologia, personagens envolvidos e manifestações clínicas sugeridas.

- Relacionar os fármacos citados no filme: para cada fármaco, deve ser elaborado um resumo do grupo farmacológico a que pertence, descrevendo: seus mecanismos de ação, principais efeitos farmacológicos e efeitos adversos. Se, durante o filme, não houver indicação de uso de fármacos, recomenda-se relacionar os fármacos que poderiam ser utilizados no tratamento da patologia implicada no filme, descrevendo seus aspectos farmacológicos.

\section{Discussão do filme}

O mesmo roteiro utilizado para elaboração do relatório também tem serventia para o debate sobre o filme na aula seguinte. O debate implica dialogar sobre os temas, permitindo que os alunos se posicionem sobre os assuntos. Nesse sentido, é importante:

- Incentivar a participação oral e estimular a participação de todos os alunos: indicar as cenas do filme que apresentam vínculo com o tema da aula pode servir de estratégia para estimular a participação dos alunos;

- Estabelecer uma relação entre a narrativa do filme e a patologia em foco, seus aspectos clínicos, e os fármacos utilizados;

- Relacionar os fármacos utilizados com a patologia implicada no filme;

- Discutir mecanismos de ação dos fármacos, farmacocinética, efeitos farmacológicos e adversos;

- Aproveitar para consolidar conceitos sobre a patologia e farmacoterapia;

- Abordar outros aspectos da terapêutica: suporte psicológico, procedimentos de Enfermagem e orientações para os pacientes e familiares;

- Debater princípios éticos envolvidos na pesquisa com seres humanos - se o assunto permitir.

O debate é finalizado elaborando-se uma síntese dos aspectos mencionados durante a discussão.

A avaliação é feita somando-se as notas dos relatórios e a participação individual nos debates.

No Quadro 1, tem-se a relação dos conteúdos teóricos e os filmes utilizados durante a disciplina Neurofarmacologia. 
Quadro 1. Relação de filmes utilizados no Curso de Neurofarmacologia - FS-UnB

\begin{tabular}{|l|l|}
\hline \multicolumn{1}{|c|}{ Tema da aula } & \multicolumn{1}{c|}{ Filme } \\
\hline 1 - Farmacologia dos anestésicos gerais & Linha mortal \\
\hline 2 - Fármacos antidepressivos e distúrbio bipolar & Mr. Jones \\
\hline 3 - Fármacos antipsicóticos: esquizofrenia & Uma mente brilhante \\
\hline 4 - Farmacologia de doenças neurodegenerativas & Óleo de Lorenzo \\
\hline 5 - Farmacologia do Parkinson & Tempo de despertar \\
\hline 6 - Farmacologia do Alzheimer & Íris \\
\hline 7 - Farmacologia da dependência e abuso de drogas & 28 dias \\
\hline
\end{tabular}

Neste artigo, serão ilustrados quatro filmes utilizados durante a disciplina, a saber: Linha Mortal, Mr. Jones, Tempo de Despertar e Uma mente Brilhante.

Filme 1: Linha mortal

Título original: Flatliners

Direção: Joel Schumacher

Elenco: Kiefer Sutherland, Julia Roberts, Kevin Bacon, William Baldwin,

Oliver Platt, Kimberly Scott, Joshua Rudoy

Produção: Rick Bieber, Michael Douglas

País de Origem: EUA

Gênero: Suspense

Tempo de duração: 115 minutos

Cor: Colorido

Ano de lançamento: 1990

Distribuidora: Columbia Home Video

Estúdio: Columbia Pictures

\section{Sinopse}

Linha mortal conta a história de cinco estudantes de Medicina que decidem fazer uma experiência ousada a fim de descobrir se existe vida após a morte. No Hospital-Escola da Faculdade de Medicina, um grupo de estudantes provoca condições que podem levá-los à morte e à sua reversão.

A experiência dá-se com um integrante do grupo por vez. Os estudantes se revezam e são induzidos a um estado bastante próximo à morte e, então, são reanimados. Cada um tenta ficar do "outro lado" o maior tempo possível. O mais visionário deles é Nelson. Ele quer provocar uma parada cardíaca em si mesmo e passar clinicamente morto durante um minuto, antes de ser reanimado pelos amigos. Seu desejo é saber se há vida após a morte. Mesmo com medo, eles levam a ideia em frente. Nelson retorna do "limbo" maravilhado com as visões que a morte artificial lhe trouxe. Instigados pelo sucesso, os demais estudantes decidem repetir, um por um, a experiência. Cada integrante do grupo passa um pouco mais de tempo do que o anterior, do "outro lado", e a linha que separa a vida e a morte começa a diminuir. Além disso, todos os que visitam o "limbo" experimentam visões sinistras. Durante a "experiência", são utilizados vários fármacos para induzir a "morte" temporária e, posteriormente, para causar a reanimação cardíaca. 
Roteiro para elaboração do resumo e para o debate do filme Linha mortal

. Relacionar os fármacos citados no filme: pentotal sódico, óxido nitroso, adrenalina, atropina, bretílio, lidocaína, cloreto de potássio;

. Fazer uma análise crítica se os fármacos e os efeitos observados no contexto do filme ou da cena analisada são condizentes com a sua utilização e seus efeitos farmacológicos;

- Explorar o modo de ação dos fármacos, farmacocinética e efeitos adversos;

- Debater sobre interação medicamentosa;

- Debater sobre os períodos e estágios da anestesia geral;

- Comentar sobre outros fármacos anestésicos gerais e uso de adjuvantes da anestesia geral ou medicação pré-anestésica;

. Neste caso, em especial, o debate pode ser conduzido para os fármacos usados na reanimação cardíaca, antiarrítmicos e equilíbrio hidroeletrolítico.

Filme 2: Mr. Jones

Título original: Mr. Jones

Diretor: Mike Figgis

Elenco: Richard Gere, Lena Olin, Anne Bancroft, Tom Irwin, Delroy Lindo

Produção: Jerry A. Baerwitz, Richard Gere

País de origem: EUA

Gênero: Romance

Tempo de duração: 114 minutos

Cor: Colorido

Ano de lançamento: 1993

Estúdio/Distrib.: Columbia Home Video

\section{Sinopse}

Mr. Jones (Richard Gere) é um homem que atrai as pessoas com o seu encanto e a sua energia vital. Entretanto, ele tem um perfil característico das pessoas acometidas por transtorno bipolar. Apresenta alternância de manifestações de comportamentos, às vezes, depressivos e, em outros momentos, de euforia demasiada.

Em uma das crises de mania, Mr. Jones chega ao local da construção de um edifício onde, alegando ser marceneiro, pede emprego. Relutante no início, o encarregado termina permitindo que ele faça uma experiência de três dias. Jones é levado até o topo da estrutura, onde um grupo de marceneiros trabalha. Em pouco tempo, vai até a extremidade de uma viga, de onde ameaça voar. Preocupado, o encarregado pede ajuda e Mr. Jones é encaminhado para um Hospital Psiquiátrico, onde a Dra. Elizabeth Bowen o diagnostica como um psicótico maníaco-depressivo, sendo, então, medicado na emergência. No dia seguinte, ao entrar num teatro, onde uma orquestra sinfônica apresenta a $9^{a}$ sinfonia de Beethoven, Jones sobe ao palco e passa a interferir no trabalho do maestro, sendo removido, então, pela polícia até o hospital. Lá, é atendido por um médico, mas pede que sua médica, a Dra. Elizabeth, seja chamada. Esta chega e lhe diz que ele está muito doente e que precisa de tratamento e de internação.

A sua psiquiatra, a Dra. Elizabeth Bowen, está decidida a ajudá-lo, mas logo estará profundamente ligada a ele.

Roteiro para elaboração do resumo e para o debate do filme Mr. Jones

. Relacionar os fármacos citados no filme: haldole amytal;

- Explorar a fisiopatologia e características clínicas dos distúrbios afetivos;

. Analisar o modo de ação do haldole amytal e outros fármacos utilizados no tratamento de distúrbios afetivos;

- Debater a associação com outros fármacos e a importância da interação medicamentosa; 
- Debater sobre a farmacocinética e efeitos adversos dos fármacos utilizados em distúrbios afetivos;

. Discutir sobre a importância da orientação do profissional de saúde a respeito dos possíveis efeitos colaterais e como o paciente deve proceder para minimizar tais manifestações;

. Discutir sobre a importância da orientação aos familiares quanto ao acompanhamento do paciente com distúrbios afetivos.

Filme 3: Tempo de despertar

Título original: Awakenings

Diretor: Penny Marshall

Elenco: Robert De Niro, Robin Williams, Julie Kavner, Ruth Nelson, John

Heard

Produção: Elliot Abbott, Elliot Abbott, Lawrence Lasker

País de origem: EUA

Gênero: Drama

Tempo de duração: 120 minutos

Cor: Colorido

Ano de lançamento: 1990

Estúdio/Distrib.: Columbia Pictures Corporation

\section{Sinopse}

O Hospital Bainbridge é uma instituição para doentes crônicos localizada no bairro do Bronx, em Nova lorque, EUA. O ano é 1969. O filme apresenta a história verdadeira do Dr. Malcolm Sayer (Robin Williams), um neurologista muito experiente como pesquisador, mas com pouco prática clínica, que conseguiu emprego na referida instituição hospitalar. Quando começa a trabalhar, o médico encontra vários pacientes que aparentemente estão catatônicos e que se encontram em tal estado desde o final da I Guerra Mundial, em decorrência de um surto da chamada doença do sono - a encefalite letárgica. Sayer observa detalhadamente aqueles pacientes e acredita que estejam apenas "adormecidos" e que poderão "despertar" se tomarem a medicação correta. Assim, o médico pesquisa minuciosamente o assunto e chega à conclusão de que a $L-D O P A$, uma nova droga que já estava sendo utilizada para pacientes com o mal de Parkinson, poderia ser benéfica em tais pacientes. No entanto, ao levar o assunto para o diretor do hospital e solicitar autorização para realizar o tratamento, consegue permissão para testá-lo em apenas um paciente. Sayer, então, escolhe Leonard Lowe (Robert De Niro) - o mais novo deles -, que há décadas estava "adormecido". Gradualmente, Lowe se recupera e isto encoraja Sayer a administrar a L-DOPA em outros pacientes, sob sua supervisão. Logo, estes apresentam sinais de melhora e se sentem ansiosos para recuperar o tempo perdido. Mas, infelizmente, Lowe começa a apresentar efeitos colaterais estranhos que não estavam previstos. O filme mostra a perda da eficácia da levodopa ( $L-D O P A)$ e o aparecimento dos efeitos adversos, como, por exemplo, a distonia. O tratamento tem de ser suspenso e, gradualmente, os pacientes voltam à sua condição inicial.

Roteiro para elaboração do resumo e para o debate do filme Tempo de despertar

- Relacionar os fármacos citados no filme: L-DOPA;

. Explorar a fisiopatologia de distúrbios neurodegenerativos, como, por exemplo, a doença de Parkinson;

. Analisar o modo de ação da L-DOPA ou levodopa e de outros fármacos utilizados no tratamento do Parkinson;

. Debater a associação com outros fármacos e a importância da interação medicamentosa;

- Debater sobre a farmacocinética e efeitos adversos dos antiparkinsonianos;

- Analisar a diferença entre tratamento terapêutico e pesquisa terapêutica;

- Debater as pesquisas clínicas e os aspectos éticos envolvidos em tais pesquisas; 
. Discutir sobre a orientação do profissional de saúde sobre os possíveis efeitos colaterais e como o paciente deve proceder para minimizar tais manifestações;

. Discutir sobre a importância da orientação aos familiares e cuidadores no acompanhamento do paciente com Parkinson.

Filme 4: Uma mente brilhante

Título original: A beautiful mind

Diretor: Ron Howard

Elenco: Russell Crowe, Jennifer Connelly, Ed Harris, Paul Bettany, Scott

Fernstrom, Josh Lucas, Ned Stuart

Produção: Brian Grazer

País de origem: EUA

Gênero: Drama

Tempo de duração: 134 minutos

Cor: Colorido

Ano de lançamento: 2001

Estúdio: Universal Pictures/DreamWorks SKG/Imagine Entertainment

\section{Sinopse}

O filme Uma mente brilhante baseia-se na biografia de John Nash, escrita por Sylvia Nasar, e começa com a chegada do jovem Nash à Universidade de Princenton, em 1947. Nash tem dificuldades de participar das atividades básicas da Universidade, como assistir às aulas e conviver com os colegas. O seu objetivo era fazer uma grande descoberta, ter uma "ideia original" e, assim, receber o reconhecimento das pessoas. Para tanto, dedicava todo o seu dia ao estudo e à investigação. Durante a faculdade, seu único amigo era um suposto colega de quarto de nome Charles, que o incentivava a procurar a sua "ideia original". Nash inspira-se em fontes estranhas, mas será numa conversa de bar que ele encontrará inspiração para a sua "ideia original" : uma teoria revolucionária sobre a economia moderna. O reconhecimento pelo seu trabalho acontece em 1953, após ter realizado alguns trabalhos no Pentágono para decifrar códigos russos. Nash também era professor e, nesse período, que ministra suas aulas, conhece uma aluna chamada Alicia, com quem viria a se casar e a ter um filho. Tudo parecia correr bem com o casal, até que Nash começa a ser perseguido por desconhecidos.

Paralelamente, Nash é chamado para executar um serviço misterioso e confidencial. Para Nash, a missão confidencial estava cada vez mais perigosa e as perseguições mais constantes. Um dia, em uma apresentação profissional, ao se ver perseguido, fugiu da sala acometido por um surto. Nash foi levado ao hospital psiquiátrico, onde foi diagnosticado com esquizofrenia paranoide. Deu-se, então, início a um tratamento, mas sob o efeito dos medicamentos, não conseguia mais ser um bom matemático. Resolveu, assim, parar de tomar os remédios, mas entrou novamente em crise, chegando a colocar a vida da esposa e do filho em risco. Logo, Nash percebe que as alucinações não eram reais e decide lutar contra a doença, mas sem voltar a usar os medicamentos. Nash conviveu com as alucinações e foi aprendendo a lidar com elas. Prosseguiu com seus estudos e as pesquisas, consagrando-se por suas descobertas. O filme termina com o reconhecimento pelo qual Nash tanto ansiava: o Prêmio Nobel de Economia em 1994, por sua contribuição com a Teoria dos Jogos.

Roteiro para elaboração do resumo e para o debate do filme Uma mente brilhante

- Relacionar os fármacos citados no filme: thorazine;

- Explorar os aspectos clínicos e fisiopatológicos dos distúrbios psicóticos como, por exemplo, a esquizofrenia;

. Analisar o mecanismo de ação dos antipsicóticos e fármacos usados no tratamento da esquizofrenia;

. Debater a associação com outros fármacos e a importância da interação medicamentosa; 
- Debater sobre a farmacocinética e efeitos adversos dos antipsicóticos;

. Discutir a importância de orientar o paciente quanto aos possíveis efeitos indesejáveis dos antipsicóticos;

. Discutir sobre a importância dos familiares e pessoas próximas no trato com o paciente esquizofrênico.

\section{Considerações finais}

A utilização de filmes como ferramenta do ensino da Neurofarmacologia tem demonstrado que, se bem trabalhadas, as películas podem ser incluídas no plano de aula dos cursos de graduação da área da saúde: Medicina, Enfermagem, Odontologia, Biomedicina, Biologia, Farmácia e Saúde Coletiva. Tal fato demanda que os responsáveis façam um planejamento e, ao escolherem os filmes, tenham o cuidado de relacioná-los aos temas que serão abordados, para que o aprendizado seja complementar e genuinamente significativo (Guilhem, Diniz, Zicker, 2007).

O uso deste recurso pedagógico requer um planejamento prévio. O filme pode ser projetado em sala de aula, seguido de debate. Pode-se, também, projetar parte do filme, interromper a projeção para fazer comentários, ou responder algumas dúvidas ou questionamentos dos estudantes. Neste caso, o debate é realizado concomitante à projeção do filme. O filme pode ainda ser utilizado como atividade extraclasse, ou seja, o debate deverá ser realizado em outra oportunidade. No curso aqui analisado, sugere-se a realização dos debates na aula seguinte.

O filme, como complemento de um conteúdo pedagógico, tem importância por ser uma forma de sensibilizar o aluno para o tema escolhido (Toman, Rak, 2000; Timm et al., 2004). Durante o curso, observou-se que nem todos os alunos conhecem a metodologia, em especial, quando o foco é a farmacologia, porém, reconhecem a relevância do emprego de filmes para aproximá-los de situações clínicas, servindo como adjuvante no esclarecimento da patologia e da terapêutica.

Segundo Timm et al. (2004), a motivação para o direcionamento da atenção do aluno é outro fator importante a ser levado em consideração, contribuindo, desta forma, para o acesso ao conteúdo. Por ser uma atividade lúdica e prazerosa, o filme prende a atenção dos discentes e ajuda no desenvolvimento das relações humanas, uma vez que o aluno pode debater com seus pares e trocar informações para aprofundamento e construção do conhecimento.

O professor, por outro lado, deve atuar como um estimulador da discussão, trazer para o debate fatos relevantes do filme, sempre direcionando a discussão para o assunto da aula (Guilhem, Diniz, Zicker, 2007). Embora o enfoque do uso de filmes seja sobre os aspectos clínicos e farmacológicos, é preciso, durante o debate, ter o cuidado da ênfase ao respeito aos pacientes e aos conceitos que cada um traz em seu conteúdo cultural, conduzindo à reflexão de novas possibilidades de pensamento. Tal fato possibilita um olhar ampliado em relação ao paciente, não apenas com base em conhecimento técnico, mas de novos saberes e condutas.

De fato, a presente discussão mostra-se relevante, em especial, por se tratarem de assuntos delicados relacionados à farmacologia de distúrbios afetivos, de distúrbios neurodegenerativos, ou a farmacologia da dependência e abuso de drogas, nos quais a atuação do profissional de saúde precisa, muitas vezes, estar não somente focada na terapêutica medicamentosa, mas na humanização e na relação entre profissional e paciente.

Pelo fato de os filmes serem utilizados como atividade prévia extraclasse, observou-se que os alunos demonstram, possivelmente, maior disposição para o debate, por possibilitar a pesquisa sobre os aspectos clínicos da doença, tratamento farmacológico e mecanismos de ação dos fármacos, conforme roteiro previamente estabelecido para discussão, permitindo-os, muitas vezes, relacionar com alguns casos clínicos observados em outras disciplinas ou durante os estágios hospitalares.

A experiência obtida com a utilização de filmes comerciais no curso de Neurofarmacologia tem apresentado grande motivação dos estudantes para o debate e contribuído para um melhor rendimento nas avaliações da referida disciplina. 


\section{Colaboradores}

Setsuko Santos responsabilizou-se pela elaboração do artigo; Andre Noro responsabilizou-se pela pesquisa dos filmes e pelos roteiros dos mesmos.

\section{Referências}

ANDERSON, D. Using feature films as tools for analysis in a psychology and law course. Teach. Psycol., v.19, n.3, p.155-8,1992.

BERGER, J.; PRATT, C. Teaching business-communication ethics with controversial films. J. Bus. Ethics, v.17, n.16, p.1817-23, 1998.

BHAGAR, H.A. Should cinema be used for medical student education in psychiatry? Med. Educ., v.39, n.9, p.972-3, 2005.

CONNER, D. From monty Python to total recall: a feature film activity for the cognitive psycology course. Teach. Psycol., v.23, n.1, p.33-5, 1996.

CRELLIN, J.K.; BRIONES, A.F. Movies in medical education. Acad. Med., v.70, n.9, p.745, 1995.

FARRÉ, M. et al. Putting clinical pharmacology in context: the use of popular movies. J. Clin. Pharmacol., v.44, n.1, p.30-6, 2004.

GALLAGHER, P. et al. A pilot study of medical student attitudes to, and use of, commercial movies that address public health issues. BMC Res. Notes, v.4, n.7, p.111, 2011.

GUILHEM, D.; DINIZ, D.; ZICKER, F. (Eds.). Pelas lentes do cinema: bioética e ética em pesquisa. Brasília: Letras Livres/EdUnB, 2007.

HYDE, N.B.; FIFE, E. Innovative instructional strategy using cinema films in an undergraduate nursing course. ABNF J., v.16, n.5, p.95-7, 2005.

KOREN, G. Awakenings: Using a popular movie to teach clinical pharmacology. Clin. Pharmacol. Ther., v.53, n.1, p.3-5, 1993.

LEPICARD, E.; FRIDMAN, K. Medicine, cinema and culture: a workshop in medical humanities for clinical years. Med. Educ., v.37, n.11, p.1039-40, 2003.

MAESTRELLI, S.R.P.; FERRARI, N. O óleo de Lorenzo: o uso do cinema para contextualizar o ensino de genética e discutir a construção do conhecimento científico. Genet. Esc., v.1, n.2, p.35-9, 2006. Disponível em: <http://

www.geneticanaescola.com.br/ano1vol2/02.pdf>. Acesso em: 7 maio 2012.

SIERLES, F.S. Using film as the basis of an american culture course for first-year psychiatry residents. Acad. Psychiatry, v.29, n.1, p.100-4, 2005.

TIMM, M.I. et al. Tecnologia educacional: apoio à representação do professor de Ciência e Tecnologia e instrumento de estudo para o aluno. RENOTE Nov. Tecnol. Educ., v.2, n.2, p.1-10, 2004.

TOMAN, S.; RAK, C. The use of cinema in the counselor education curriculum: strategies and outcomes. Couns. Educ. Superv., v.40, n.2, p.105-14, 2000. 
Recursos audiovisuais são estratégias utilizadas para facilitar o processo ensino-aprendizagem. Entretanto, o uso de filmes comerciais como recurso pedagógico para o ensino das ciências da saúde, tal como a farmacologia, não é tão comum. O objetivo deste artigo é relatar nossa experiência com o uso de filmes, como: Linha Mortal, Mr. Jones, Tempo de despertar, entre outros, como ferramenta de ensino da disciplina Neurofarmacologia. Embora seja ministrada para o ensino de graduação do curso de Enfermagem da Universidade de Brasília, a metodologia pode ser adotada em outros cursos da área de Ciências da Saúde. A combinação de referencial teórico de Neurofarmacologia e a discussão dos filmes em sala de aula pode ser comparada a um estudo de caso, cuja narrativa permite aproximar os estudantes da realidade, tornando lúdico e fácil de relacionar as situações clínicas com o tratamento farmacológico, além de assimilarem novos conceitos.

Palavras-chave: Cinema como assunto. Recursos audiovisuais. Neurofarmacologia.

\section{The use of films as an educational resource on neuropharmacology teaching}

Audiovisual resources are strategies used to facilitate the process of teaching. However, the use of commercial films as an educational resource for teaching health sciences, such as pharmacology, is not very common. The purpose of this article is to describe our experience with using films, as Flatliners, Mr. Jones, Awakenings, among others, as a teaching tool for the Neuropharmacology course. Although it is used to teach undergraduates at the Nursing course of Brasilia University, the methodology can be adopted by other courses in the field of Health Sciences. The combination of the theoretical framework of Neuropharmacology and the classroom discussion of the films can be compared to a case study, whose narrative allows the students to be brought closer to reality, making it entertaining and easier for them to relate to clinical situations, with pharmacotherapy, and to assimilate new concepts.

Keywords: Motion pictures as topic. Audiovisual resource. Neuropharmacology.

\section{El uso de películas como recurso pedagógico en la enseñanza de neurofarmacología}

Los recursos audiovisuales son estrategias para facilitar el proceso de enseñanza-aprendizaje. El uso de películas comerciales como recurso pedagógico para la enseñanza de las ciencias de la salud, tal como la farmacología, no es tan común. El objetivo de este artículo es relatar nuestra experiencia al usar películas, como, Línea Mortal, Mr. Jones, Tiempo de despertar, entre otras, como herramienta de enseñanza de la asignatura de Neurofarmacología. Aunque se utilice para la enseñanza del curso de graduación de Enfermería de la Universidad de Brasilia, la metodología se puede adoptar en otros cursos de las Ciencias de la Salud. La combinación del referencial teórico de Neurofarmacología y la discusión de las películas en clases se puede comparar a un estudio del caso, cuya narrativa permite aproximar los estudiantes a la realidad, volviendo lúdico y más fácil relacionar las situaciones clínicas con el tratamiento farmacológico y assimilar nuevos conceptos.

Palabras clave: Cine como assunto. Medios audiovisuales. Neurofarmacología. 